\title{
ON STRATIFIED MUKAI FLOPS
}

\author{
Pierre-Emmanuel Chaput and Baohua Fu
}

\section{Introduction}

In recent studies (see [14], [6]) of birational geometry of symplectic resolutions of nilpotent orbit closures, three types of flops (which will be called stratified Mukai flops of type $A, D, E_{6}$ ) are shown to be fundamental, in the sense that others can be decomposed into a sequence of these flops. Stratified Mukai flops of type $A$ are given by cotangent bundles of dual Grassmanians $T^{*} G(k, V) \rightarrow T^{*} G\left(k, V^{*}\right)$, which has been previously studied by Markman([12]). The type $D$ stratified Mukai flop is given by the cotangent bundles of the two connected components of the orthogonal Grassmannian $G_{i s o}(k, 2 k)$, where $k$ is an odd integer. There are two stratified Mukai flops of type $E_{6}$, corresponding to the pairs of roots $\left(\alpha_{1}, \alpha_{6}\right)$ (type $\left.E_{6, I}\right)$ and $\left(\alpha_{3}, \alpha_{5}\right)$ (type $\left.E_{6, I I}\right)$.

For a stratified Mukai flop $\mu: T^{*}(G / P) \rightarrow T^{*}(G / Q)$, an important problem is to construct explicitly a $G$-equivariant equivalence between the derived categories of $T^{*}(G / P)$ and $T^{*}(G / Q)$ which is compatible with the flop $\mu$. It is tempting to believe that a Fourier-Mukai transform with kernel induced by a suitable resolution (possibly with several irreducible components) of this flop could yield such an equivalence.

In [2] and [6], we showed that the graph of the stratified Mukai flop is in fact smooth, thus it gives a resolution of the flop. Although very natural, this resolution does not lead to easy computations in Chow groups nor in the derived categories. Our first result (Theorem 1, which works also for type $D$ Mukai flops) of this note is to resolve the stratified Mukai flop of type $E_{6, I}$ by blow-ups along smooth centers. A similar resolution has already been constructed by Markman [12] for stratified Mukai flops of type $A$. As we will see, our resolution is constructed in a very similar way, blowing up successively the different $G$-orbits, from the smallest to the biggest.

Like the usual Mukai flops, this resolution does not induce an isomorphism between the Chow groups (cf. Example 1, Example 2). To find out a natural isomorphism between the Chow groups, we will first construct a deformation of this flop, then we will show that the deformed flop can be resolved in a similar way. Using a beautiful idea of [11], we prove that this leads to a natural isomorphism between Chow groups and their Chow motifs. This shows that the stratified Mukai flop of type $E_{6, I}$ is very similar to the usual Mukai flop. Unfortunately, we do not know if this functor also gives an equivalence between derived categories.

Received by the editors December 11, 2006.

2000 Mathematics Subject Classification. 14L30, 14M17, $20 \mathrm{G} 15$.

Key words and phrases. stratified Mukai flops, blow-ups, Chow groups. 


\section{The wonderful resolution}

Let $G$ be a simple group of type $A_{n}, D_{2 n+1}$ or $E_{6}$. Let $(P, Q)$ be the maximal parabolic subgroups corresponding respectively to the roots $\left(\alpha_{i}, \alpha_{n+1-i}\right)$ with $2 i<$ $n+1,\left(\alpha_{2 n}, \alpha_{2 n+1}\right)$ and $\left(\alpha_{1}, \alpha_{6}\right)$.

Set $X_{P}=G / P$ and $T_{P}=T^{*}(G / P)$. We denote by $\pi_{P}: T_{P} \rightarrow X_{P}$ the natural projection. We know that the order on the set of $G$-orbits in $T_{P}$ given by $\mathcal{O} \leq \mathcal{O}^{\prime}$ if $\mathcal{O} \subset \overline{\mathcal{O}^{\prime}}$ is a total order, so we choose a labelling $T_{P}^{i}, 0 \leq i \leq r$ of the $G$-orbits in $T_{P}$ such that $T_{P}^{i} \subset \overline{T_{P}^{j}}$ if and only if $i \leq j$. Note that $r=i$ in type $A_{n}$ with $P, Q$ corresponding to $\alpha_{i}, \alpha_{n+1-i}$, and that $r$ equals $n$ in type $D_{2 n+1}$ and 2 in type $E_{6}$. Similar notations will be used for the parabolic subgroup $Q$. It is known that there exists a birational map $\mu: T_{P} \rightarrow T_{Q}$, which will be called a stratified Mukai flop. Note that $\mu$ is an isomorphism between the open orbits $T_{P}^{r} \rightarrow T_{Q}^{r}$.

We consider successive blow-ups defined recursively as follows. Let $B l_{0}\left(T_{P}\right):=T_{P}$ and $B l_{0}\left(\overline{T_{P}^{i}}\right)=\overline{T_{P}^{i}}$. For $1 \leq k \leq r$ an integer, we let $B l_{k}\left(T_{P}\right)$ be the blow-up of $B l_{k-1}\left(T_{P}\right)$ along $B l_{k-1}\left(\overline{T_{P}^{k-1}}\right), B l_{k}\left(\overline{T_{P}^{k-1}}\right)$ the exceptional divisor of this blow-up and $B l_{k}\left(\overline{T_{P}^{i}}\right)(i \neq k-1)$ the proper transform of $B l_{k-1}\left(\overline{T_{P}^{i}}\right)$ under this blow-up. We set $\widetilde{T}_{P}:=B l_{r}\left(T_{P}\right)$ and $\widetilde{T}_{P}^{i}:=B l_{r}\left(\overline{T_{P}^{i}}\right)$. Note that the natural blow down map restricts to an isomorphism over the open $G$-orbit. We also consider the analogous construction for $Q$.

Theorem 1. Under the above notations, we have

(i) The varieties $B l_{k}\left(\overline{T_{P}^{k}}\right), B l_{k}\left(T_{P}\right)$ are smooth for any $k$. The divisors $\widetilde{T}_{P}^{i}(i=$ $0, \cdots, r-1)$ are smooth irreducible with normal crossing inside $\widetilde{T}_{P}$ and Pic $\left(\widetilde{T}_{P}\right) \simeq$ $\operatorname{Pic}(G / P) \oplus_{i=0}^{r-1} \mathbb{Z}\left[\widetilde{T}_{P}^{i}\right]$;

(ii) Two points $x, y \in \widetilde{T}_{P}$ belong to the same $G$-orbit if and only if $\left\{i \mid x \in \widetilde{T}_{P}^{i}\right\}=$ $\left\{j \mid y \in \widetilde{T}_{P}^{j}\right\}$;

(iii) There is a unique G-equivariant isomorphism $\widetilde{\mu}: \widetilde{T}_{P} \simeq \widetilde{T}_{Q}$ such that $\widetilde{\mu}$ identifies with $\mu$ over the open $G$-orbit.

Remark 1. 1). Claims (i) and (ii) are some properties of wonderful compactifications of symmetric spaces ([4]). This is why we called this resolution wonderful.

2). We do not know if a similar result holds for stratified Mukai flop of type $E_{6, I I}$. In this case, the inclusion relationship of orbit closures in $T^{*} X_{P}$ is no longer linear.

We will need the following lemma in the proof of the theorem.

Lemma 1. Let $Y$ be a variety and assume there are morphisms $t_{P}: Y \rightarrow T_{P}, x_{Q}$ : $Y \rightarrow X_{Q}$ such that $t_{P}^{-1}\left(T_{P}^{r}\right)$ is dense in $Y$ and $\forall y \in t_{P}^{-1}\left(T_{P}^{r}\right), \pi_{Q} \circ \mu \circ t_{P}(y)=x_{Q}(y)$. Then there is a unique morphism $t_{Q}: Y \rightarrow T_{Q}$ which lifts $x_{Q}$ via $\pi_{Q}$ and such that $\forall y \in t_{P}^{-1}\left(T_{P}^{r}\right), \mu \circ t_{P}(y)=t_{Q}(y)$.

Proof : The uniqueness of $t_{Q}$ is clear. Now we prove the existence following [2]. A pair $(p, q) \in X_{P} \times X_{Q}$ is called incident if $\operatorname{Stab}(p) \cap \operatorname{Stab}(q)$ is a parabolic subgroup of $G$. For $p \in X_{P}$, let $C_{p} \subset X_{Q}$ denote the set of points incident to $p$, and define similarly $C_{q}$, for $q \in X_{Q}$. For an incident pair $(p, q)$, recall that there is a welldefined linear isomorphism $\mu(p, q): T_{p} X_{P} / T_{p} C_{q} \rightarrow T_{q} X_{Q} / T_{q} C_{p}$ [2, theorem 4.1]. Let $\left(T_{q} C_{p}\right)^{\perp}$ be the subspace of $T_{q}^{*} X_{Q}$ consisting of co-vectors vanishing identically 
on $T_{q} C_{p}$. Similarly, we get $\left(T_{p} C_{q}\right)^{\perp}$. The transposed map ${ }^{t} \mu(p, q):\left(T_{q} C_{p}\right)^{\perp} \rightarrow$ $\left(T_{p} C_{q}\right)^{\perp}$ is then again an isomorphism. Furthermore by [2, theorem 4.1], for any $t \in\left(T_{p} C_{q}\right)^{\perp} \cap T_{P}^{r}$, we have $\mu(t)=\left(q,{ }^{t} \mu(p, q)^{-1}(t)\right)$.

Note that for any $y \in Y$, the pair $p:=\pi_{P} \circ t_{P}(y)$ and $q:=x_{Q}(y)$ are incident. In fact, this is true if $y \in t_{P}^{-1}\left(T_{P}^{r}\right)$ (see for example [2, theorem 3.3]). Now the density of $t_{P}^{-1}\left(T_{P}^{r}\right)$ implies that this holds for all $y \in Y$. We claim that for any $y \in Y$, $t_{P}(y) \in\left(T_{p} C_{q}\right)^{\perp}$. In fact, if $y \in t_{P}^{-1}\left(T_{P}^{r}\right)$, then $t_{P}(y) \in T_{p}^{*} X_{P} \cap T_{q}^{*} X_{Q}=\mathfrak{p}^{\perp} \cap \mathfrak{q}^{\perp} \simeq$ $\left(T_{p} C_{q}\right)^{\perp}$, where $\mathfrak{p}$ and $\mathfrak{q}$ are the Lie subalgebras of $\operatorname{Stab}(p)$ and $\operatorname{Stab}(q)$. Again the claim follows from the density of $t_{P}^{-1}\left(T_{P}^{r}\right)$. Therefore, to conclude the proof of the lemma, it is enough to set $x_{P}(y):=\pi_{P} \circ t_{P}(y)$ and define

$$
t_{Q}(y):=\left(x_{Q}(y),{ }^{t} \mu\left(x_{P}(y), x_{Q}(y)\right)^{-1}\left(t_{P}(y)\right)\right) .
$$

Proof of the theorem : The uniqueness of $\widetilde{\mu}$ follows from the fact that $\widetilde{T}_{P}^{r}$ is dense in $\widetilde{T}_{P}$, so let us prove the existence of $\widetilde{\mu}$. Our proof is in three steps : first we give a description of $\widetilde{T}_{P}$ in terms of complete collineations; from this description we then deduce the existence of a morphism $\widetilde{T}_{P} \rightarrow \widetilde{T}_{Q}$. Finally, we show that this morphism and the analogous morphism $\widetilde{T}_{Q} \rightarrow \widetilde{T}_{P}$ are inverses one to the other. This method is very similar to that of Markman ([12]), where he proved the theorem for stratified Mukai flops of type A. We will give a complete proof in the case of $E_{6}$, and explain how this adapts to type $D$.

So consider first the case $G=E_{6}$. Then a Levi factor $L$ of $P$ is a semi-direct product of $\mathbb{C}^{*}$ and $S_{p i n}$, and if $x$ denotes the point in $G / P$ with stabilizer $P$, the representation $T_{x}^{*} X_{P}$ of $L$ is a (16-dimensional) spin representation [5, theorem 2.10]. We will denote this $L$-representation by $\mathbb{S}_{+}$.

Let $W$ be the natural 10-dimensional $L$-representation and $\mathcal{Q} \subset \mathbb{P} W$ the corresponding smooth quadric. The variety $\mathbb{P S}_{+}$has two $L$-orbits and the closed one will be denoted by $X_{+}$, which is naturally isomorphic to one component of the Grassmanian of maximal isotropic vector subspaces in $W$. Let $\widehat{X}_{+} \subset \mathbb{S}_{+}$be the cone of $X_{+}$.

Recall that there is an $L$-equivariant quadratic map $\nu_{2}: \mathbb{S}_{+} \rightarrow W$ with $\nu_{2}^{-1}(0)=$ $\widehat{X}_{+}$and that the image is contained in the affine cone of $Q$. This gives a rational map $\overline{\nu_{2}}: \mathbb{P S}_{+} \rightarrow$ Q. In fact, let $W=N \oplus P$ be an orthogonal decomposition of $W$ into isotropic subspaces; by [3, III, p.134], $\mathbb{S}_{+}$is defined as the subalgebra $C_{N}$ of $C$, the Clifford algebra of $W$, generated by $N$. Let $f \in C_{P}$ be the product of basis elements of $P$ and $\alpha: C \rightarrow C$ the "main antiautomorphism" [3, II, p.102]. By [3, II 1.6, p.105], there is a natural identification of $C$ with $\wedge W$. For $u \in C$, let $\beta(u)$ denote the image under this identification of $u f \alpha(u)$. Then $\nu_{2}$ is defined as the degree- 1 coordinate of the restriction of $\beta$ to $\mathbb{S}_{+}$. By the symmetry properties of $\beta[3, \mathrm{p} .155], \beta(u)$ allways belong to $W \oplus \wedge{ }^{9} W \subset \wedge W$, thus the fact that $\nu_{2}^{-1}(0)$ equals $\widehat{X}_{+}$, that is the set of pure spinors, follows from [3, III 3.2, p.149 and III 4.3, p.156]. Thanks to [2, proposition 1.4 and its proof], one has an easy formula for $\nu_{2}$ in terms of complexified octonions : one identifies $\mathbb{S}_{+}$with $\mathbb{O}_{\mathbb{C}} \oplus \mathbb{O}_{\mathbb{C}}$ and $W$ with $\mathbb{C} \oplus \mathbb{O}_{\mathbb{C}} \oplus \mathbb{C}$, and one has the formula

$$
\nu_{2}\left(z_{1}, z_{2}\right)=\left(z_{1} \overline{z_{1}}, z_{1} \overline{z_{2}}, z_{2} \overline{z_{2}}\right) .
$$


(therefore $\nu_{2}$ is a kind of octonionic Veronese morphism of degree 2).

Let us denote by $C S_{+}$the variety of "complete 10-dimensional spinors" defined as the graph closure of the rational map $\overline{\nu_{2}}$. This name comes from the cases $A, D$. By the following lemma $3, C S_{+} \rightarrow \mathbb{P S}_{+}$is isomorphic to the blow-up $\phi: B l_{X_{+}}\left(\mathbb{P S}_{+}\right) \rightarrow \mathbb{P S}_{+}$ of $\mathbb{P S}_{+}$along $X_{+}$.

Let $B l_{1}\left(\mathbb{S}_{+}\right) \rightarrow \mathbb{S}_{+}$be the blow-up of $\mathbb{S}_{+}$along the origin, then the exceptional fiber is $\mathbb{P S}_{+}$and $B l_{1}\left(\mathbb{S}_{+}\right)$is the total space of the tautological line bundle $\mathcal{O}(-1)$ over $\mathbb{P S}_{+}$. The strict transform of $\widehat{X}_{+}$is then the total space of the line bundle $\left.\mathcal{O}(-1)\right|_{X_{+}}$, which is smooth. Claims (i) and (ii) of the theorem follow immediately. Let $B l_{2}\left(\mathbb{S}_{+}\right)$be the blow-up of $B l_{1}\left(\mathbb{S}_{+}\right)$along the strict transform of $\widehat{X}_{+}$. Then the above discussions give that $B l_{2}\left(\mathbb{S}_{+}\right)$is just the total space of the line bundle $\phi^{*} \mathcal{O}(-1)$ over $B l_{X_{+}}\left(\mathbb{S}_{+}\right) \simeq C S_{+}$. The second graph projection gives a morphism $C S_{+} \rightarrow \mathcal{Q}$, thus a morphism $\phi^{*} \mathcal{O}(-1) \rightarrow$ Q. This is the local model, and the globalization can be obtained as follows.

By the Bruhat decomposition theorem, all homogeneous spaces (such as $X_{P}$ ) are covered by affine spaces, and thus are locally isomorphic with their tangent spaces. In our case, we thus have an isomorphism $U_{P} \simeq \mathbb{S}_{+}^{*}$ (where $U_{P} \subset X_{P}$ is a suitable open subset). We deduce that that the restriction of $T^{*} X_{P}$ to $U_{P}$ is isomorphic with $\mathbb{S}_{+}^{*} \times \mathbb{S}_{+}$. Moreover, this isomorphism is $L$-equivariant.

By [9], there are three $L$-orbits in $\mathbb{S}_{+}$, which give via this isomorphism the intersection of the three $G$-orbits in $T^{*} X_{P}$ with $T^{*} U_{P}$. Therefore, above $U_{P}$, the closure of the $G$-orbits are trivial fibrations, and to understand the result of the successive blow-ups, it is enough to understand this in one fiber.

Recall from [2] that $X_{P}$ has a minimal equivariant projective embedding $X_{P} \subset$ $\mathbb{P} V$, with $\operatorname{dim} V=27$, and that $X_{Q}$ naturally embeds in $\mathbb{P} V^{*}$. In terms of this embedding, $W$ is a linear subspace of $V^{*}$ (in fact, it is the orthogonal of the 17dimensional affine tangent space $\widehat{T_{x} X_{P}} \subset V$ ), and $\mathcal{Q}=X_{Q} \cap \mathbb{P} W \subset \mathbb{P} W$. Recall that $L$ is a Levi subgroup of $P$, so we can regard $W$ as a $P$-representation. Now consider the vector bundle $G \times{ }^{P} W$ over $X_{P}$ (it is in fact the conormal bundle of $X_{P}$ in $\mathbb{P} V$ ) and the relative quadric over $X_{P}$ defined by $G \times{ }^{P}$ Q. The previous construction gives a map $\widetilde{T}_{P} \rightarrow G \times^{P}$ Q over $X_{P}$. We have a natural inclusion $G \times{ }^{P} Q \rightarrow G \times{ }^{P}(G / Q) \simeq G / P \times G / Q$. The second projection of the latter yields our desired morphism $\widetilde{T}_{P} \rightarrow X_{Q}$. Its restriction to each fiber, considered as a rational map, is an $L$-equivariant rational map from a projectivised spinor representation of $L$ to the 8 -dimensional projective smooth quadric; since by [2, proposition 1.5$]$, there is only one such equivariant rational map, it coincides with $\pi_{Q} \circ \mu$.

By lemma 1 and [2, theorem 4.1], this morphism lifts to a morphism $\widetilde{T}_{P} \rightarrow T_{Q}$. Assume that it lifts further to a $G$-equivariant morphism $\widetilde{T}_{P} \rightarrow B l_{k-1}\left(T_{Q}\right)$ for some $k \in\{1,2\}$, then one checks that the preimage of $B l_{k-1}\left(\overline{T_{Q}^{k-1}}\right)$ is a divisor, which implies that this morphism lifts to a $G$-equivariant morphism $\widetilde{T}_{P} \rightarrow B l_{k}\left(T_{Q}\right)$. We thus get a morphism $\widetilde{\mu}: \widetilde{T}_{P} \rightarrow \widetilde{T}_{Q}$. We have already checked that the restriction of $\widetilde{\mu}$ to $\widetilde{T}_{P}^{r} \simeq T_{P}^{r}$ to $\widetilde{T}_{Q}^{r} \simeq T_{Q}^{r}$ identifies with the flop $\mu$. 
We now consider the analogous morphism $\widetilde{\mu}_{Q}: \widetilde{T}_{Q} \rightarrow \widetilde{T}_{P}$. Since it also coincides with the Mukai flop on the open orbit, it follows that $\widetilde{\mu}_{Q} \circ \widetilde{\mu}: \widetilde{T}_{P} \rightarrow \widetilde{T}_{P}$ is the identity on the open orbit; therefore it is the identity. So the theorem is proved in type $E_{6}$.

We finally explain how this proof adapts to the type $D_{2 n+1}$. Assume that $G$ is of type $D_{2 n+1}$. Let $\mathbb{C}^{4 n+2}$ be equipped with a non-degenerate quadratic form. Let $\alpha \in G / P$ and $L_{\alpha}$ the corresponding maximal isotropic subspace. As before, the fiber $\widetilde{T}_{\alpha}$ of $\widetilde{T}_{P} \rightarrow X_{P}$ over $\alpha$ is the result of the successive blowups of $T_{\alpha}(G / P) \simeq \wedge^{2} L_{\alpha}$ along the strict transforms of the $P$-orbits in $T_{\alpha}(G / P)$. Recall that the space of complete skew-symmetric forms in $L_{\alpha}^{*}$ is the graph of the rational morphism

$$
\begin{array}{rll}
\mathbb{P}\left(\wedge^{2} L_{\alpha}\right) & -\rightarrow & \mathbb{P}\left(\wedge^{2} L_{\alpha}\right) \times \mathbb{P}\left(\wedge^{4} L_{\alpha}\right) \times \cdots \times \mathbb{P}\left(\wedge^{2 n} L_{\alpha}\right) \\
{[\omega]} & \mapsto & \left([\omega],[\omega \wedge \omega], \ldots,\left[\omega^{\wedge n}\right]\right)
\end{array}
$$

By the following proposition 1, $\widetilde{T}_{\alpha}$ identifies with the total space of a line bundle over the space of complete skew-symmetric forms in $L_{\alpha}^{*}$.

Now, a point in $\widetilde{T}_{\alpha}$ therefore defines an element $\left(x_{1}, \ldots, x_{n}\right) \in \mathbb{P}\left(\wedge^{2} L_{\alpha}\right) \times \mathbb{P}\left(\wedge^{2} L_{\alpha}\right) \times$ $\cdots \times \mathbb{P}\left(\wedge^{2 n} L_{\alpha}\right)$ in the set of complete skew-symmetric forms in $L_{\alpha}^{*}$. The last element $x_{n}$ belongs to $\mathbb{P}\left(\wedge^{2 n} L_{\alpha}\right)=\mathbb{P} L_{\alpha}^{*}$; thus it defines a hyperplane in $L_{\alpha}$. Since there is a unique maximal isotropic subspace parameterized by an element in $X_{Q}$ and meeting $L_{\alpha}$ along this hyperplane, there is a natural morphism $\widetilde{T}_{\alpha} \rightarrow X_{Q}$. Then we can apply Lemma 1 and the previous arguments to conclude.

We now turn to the proof of the lemma used in the previous proof. Recall that we defined

$$
C S_{+}:=\overline{\left\{\left([s],\left[\nu_{2}(s)\right]\right) \mid s \in \mathbb{S}_{+}-\widehat{X}_{+}\right\}} \subset \mathbb{P S}_{+} \times \mathcal{Q},
$$

where $\nu_{2}: \mathbb{S}_{+} \rightarrow W$ is a $\operatorname{Spin}_{10}$-equivariant quadratic map.

Recall that there exists a unique symmetric bilinear map $\widetilde{\nu}_{2}: \mathbb{S}_{+} \times \mathbb{S}_{+} \rightarrow W$ such that $\widetilde{\nu}_{2}(s, s)=2 \nu_{2}(s)$. Recall that $W$ is the natural 10-dimensional reprenstation of $\operatorname{Spin}_{10}$; it is therefore equipped with a scalar product that we denote by $\langle\cdot, \cdot\rangle$.

Lemma 2. The variety $C S_{+}$coincides with the variety

$$
C:=\left\{([s],[x]) \in \mathbb{P S}_{+} \times \mathcal{Q} \mid \forall t \in \mathbb{S}_{+},\left\langle\widetilde{\nu}_{2}(s, t), x\right\rangle=0\right\} .
$$

Proof : For $s \in \mathbb{S}_{+}-\widehat{X}_{+}$, let $x=\nu_{2}(s) \in \widehat{Q}$. For any $t \in \mathbb{S}_{+}$, we have $\nu_{2}(s+t \epsilon) \in \widehat{Q}$ for all $\epsilon$. Thus $\left.\frac{d}{d \epsilon}\right|_{\epsilon=0} \nu_{2}(s+t \epsilon)=\widetilde{\nu}_{2}(s, t) \in \widehat{T_{x} \mathcal{Q}}=x^{\perp}$, thus $C S_{+} \subset C$. On the other hand, the projection morphism $C \rightarrow \mathcal{Q}$ is a locally trivial bundle with projective spaces as fibers, so $C$ is irreducible. Note that if $([s],[x]) \in C$ with $\nu_{2}(s) \neq 0$, then $\left[\nu_{2}(s)\right]=[x]$ because $\operatorname{Im}\left(\widetilde{\nu}_{2}(s, \cdot)\right)=x^{\perp}$. This shows that over $\mathbb{P S}_{+}-X_{+}$, the two irreducible projective varieties $C S_{+}$and $C$ coincide. As $C S_{+} \subset C$, we have the equality.

Lemma 3. $C S_{+} \simeq B l_{X_{+}}\left(\mathbb{P S}_{+}\right)$.

Proof : By lemma 2, $C S_{+}$is the total space of a locally trivial fibration with projective spaces as fibers, thus $C S_{+}$is a smooth variety. Moreover, the fiber over a point $[s] \in X_{+}$of the graph projection $C S_{+} \rightarrow \mathbb{P S}_{+}$is then isomorphic to $\mathbb{P} \operatorname{Im}\left(\widetilde{\nu}_{2}(s,).\right) \simeq \mathbb{P}^{4}$. 
From this, we see that the preimage of $X_{+}$in $C S_{+}$is a divisor. By the universal property of blow-ups, it follows that there is a morphism $f: C S_{+} \rightarrow B l_{X_{+}}\left(\mathbb{P S}_{+}\right)$. Note that the kernel of $\widetilde{\nu}_{2}(s,$.$) is the affine tangent space \widehat{T_{[s]} X_{+}}$, and $f$ restricts to an isomorphism between $\mathbb{P} \operatorname{Im}\left(\widetilde{\nu}_{2}(s,).\right)$ and $\mathbb{P}\left(\mathbb{S}_{+} / \widehat{T_{[s]} X_{+}}\right)$. Therefore, $f$ is a bijective birational morphism. By Zariski's main theorem, it is an isomorphism.

We now turn to the case of complete skew-forms. Let $n$ be an integer, and let $V$ be a vector space of dimension $2 n$ or $2 n+1$.

\section{Definition 1.}

- The variety of complete skew-forms on $V$ is the closure of the graph (denoted by $\overline{\mathbb{P} \wedge^{2} V^{*}}$ of the rational map

$$
\begin{array}{rll}
\psi: \mathbb{P}\left(\wedge^{2} V^{*}\right) & \rightarrow & \mathbb{P}\left(\wedge^{4} V^{*}\right) \times \cdots \times \mathbb{P}\left(\wedge^{2 n} V^{*}\right) \\
{[\omega]} & \mapsto & \left([\omega \wedge \omega], \ldots,\left[\omega^{\wedge n}\right]\right) .
\end{array}
$$

- We denote by $B l\left(\mathbb{P} \wedge^{2} V^{*}\right)$ the variety obtained by blowing up successively the strict transforms of the different $S L(V)$-orbits in $\mathbb{P} \wedge^{2} V^{*}$, from the smallest to the biggest.

Proposition 1. There is an $S L(V)$-equivariant isomorphism $\overline{\mathbb{P} \wedge^{2} V^{*}} \simeq B l\left(\mathbb{P} \wedge^{2} V^{*}\right)$.

Proof : This result on such a classical subject should be known to specialists. We include a short proof here, taking advantage of the recursive nature of complete skew forms, only because we were not able to find it in the literature. In [15, theorem 11.1], a similar result is proved, but it is not precisely what we need.

We first show by induction that $\overline{\mathbb{P} \wedge^{2} V^{*}}$ and $B l\left(\mathbb{P} \wedge^{2} V^{*}\right)$ are smooth, and that the pre-image of each orbit in $\mathbb{P} \wedge^{2} V^{*}$ is an irreducible divisor. We choose a subspace $L \subset V$ of dimension 2. Let $U$ denote the open subset of $\mathbb{P} \wedge^{2} V^{*}$ of elements $\left[\omega^{\prime}\right]$ such that $\omega_{\mid L}^{\prime} \neq 0$. Given $\left[\omega^{\prime}\right] \in U$, let $S_{\left[\omega^{\prime}\right]}$ be the orthogonal of $L$ in $V$ with respect to $\omega^{\prime}$, which defines a morphism $p: U \rightarrow G(\operatorname{dim} V-2, V)$. Let $q: \overline{\mathbb{P} \wedge^{2} V^{*}} \rightarrow \mathbb{P} \wedge^{2} V^{*}$ be the natural projection and $g: q^{-1}(U) \rightarrow G(\operatorname{dim} V-2, V)$ the composition. Fix an element $S_{\left[\omega^{\prime}\right]} \in G(\operatorname{dim} V-2, V)$, then $V=L \oplus S_{\left[\omega^{\prime}\right]}$. By definition, $g^{-1}\left(S_{\left[\omega^{\prime}\right]}\right)=$ $\left\{\left([\omega], \cdots,\left[\omega^{\wedge n}\right]\right) \mid \omega \in \wedge^{2} V^{*}, \omega\left(L, S_{\left[\omega^{\prime}\right]}\right)=0\right\}$.

We fix a non-zero element $\lambda \in \wedge^{2} L^{*}$. Any element $[\omega]$ such that $\omega\left(L, S_{\left[\omega^{\prime}\right]}\right)=0$ can be uniquely written as the class of $\lambda+\omega_{0}$, with $\omega_{0} \in \wedge^{2} S_{\left[\omega^{\prime}\right]}^{*}$. Note that $\left(\lambda+\omega_{0}\right)^{\wedge i}=$ $\omega_{0}^{\wedge i}+i \lambda \wedge \omega_{0}^{\wedge(i-1)}$. From this one can deduce that $g^{-1}\left(S_{\left[\omega^{\prime}\right]}\right)$ is isomorphic to the total space of a line bundle $L$ over $\overline{\left\{\left(\left[\omega_{0}\right], \cdots,\left[\omega_{0}^{\wedge n-1}\right]\right) \mid \omega_{0} \in \wedge^{2} S_{\left[\omega^{\prime}\right]}^{*}\right\}}$, namely the pull-back of the tautological line bundle on $\mathbb{P} \wedge^{2} S_{\left[\omega^{\prime}\right]}^{*}$. Thus $g: q^{-1}(U) \rightarrow G(\operatorname{dim} V-2, V)$ is a fibration with fiber $L$. Thus the claim follows from induction. Similar arguments prove the claim for $B l\left(\mathbb{P} \wedge^{2} V^{*}\right)$.

We have a surjective morphism $f: \overline{\mathbb{P} \wedge^{2} V^{*}} \rightarrow B l\left(\mathbb{P} \wedge^{2} V^{*}\right)$ by the universal property of blow-ups. By Zariski's main theorem, $f$ has connected fibers, thus every exceptional divisor of the morphism $\overline{\mathbb{P} \wedge^{2} V^{*}} \rightarrow \mathbb{P} \wedge^{2} V^{*}$ is mapped birationally to an exceptional divisor of $B l\left(\mathbb{P} \wedge^{2} V^{*}\right) \rightarrow \mathbb{P} \wedge^{2} V^{*}$, which gives that $\operatorname{Exc}(f)$ has codimension $\geq 2$ if 
non-empty. As $f$ is a birational map between two smooth varieties, thus $\operatorname{Exc}(f)$ is empty and $f$ is an isomorphism.

We will now study in more detail the case of $G=E_{6}$, in particular, we will give another more geometric proof of our theorem in this case. Let $Z$ denote the graph closure of the flop $T_{P} \rightarrow T_{Q}$ and $R=P \cap Q$ the standard parabolic subgroup corresponding to the roots $\alpha_{1}, \alpha_{6}$. We denote by $\mathfrak{u}(P), \mathfrak{u}(Q)$ the nilradical of the Lie algebras of $P$ and $Q$ respectively. Let $\mathfrak{n}:=\mathfrak{u}(P) \cap \mathfrak{u}(Q)$. Then $\mathfrak{n}$ is an 8-dimensional $R$-representation and it is shown in [6, Theorem 6.1] that the graph closure $Z$ is isomorphic to $G \times^{R} \mathfrak{n}$. Furthermore the first graph projection $Z \rightarrow T_{P}$ is isomorphic to the composition of natural maps $G \times^{R} \mathfrak{n} \simeq G \times^{P}\left(P \times^{R} \mathfrak{n}\right) \rightarrow G \times^{P}(P \cdot \mathfrak{n})$. In particular, $P \cdot \mathfrak{n}=\mathfrak{u}(P)$ is naturally identified to $\mathbb{S}_{+}$.

Lemma 4. The natural map $\eta: P \times^{R} \mathbb{P n} \rightarrow \mathbb{P S}_{+}$is the blow-up $\phi: B l_{X_{+}}\left(\mathbb{P S}_{+}\right) \rightarrow \mathbb{P S}_{+}$ of $\mathbb{P S}_{+}$along $X_{+}$. The natural map $G \times \times^{R} \mathbb{P} \mathfrak{n} \rightarrow G \times \times^{P} \mathbb{P}(\mathfrak{u}(P))$ is the blow-up of $G \times{ }^{P} \mathbb{P}(\mathfrak{u}(P))$ along the unique $G$-closed orbit, say $O_{P}$.

Proof : First we study the $R$-representation $\mathfrak{n}$. Let $L$ be a Levi factor of $R$. As one can see on the Dynkin diagram, $L$ contains $\operatorname{Spin}_{8}$, so let $M \simeq \operatorname{Spin}_{8}$ be a subgroup of $L$. We consider an element $x$ in the root space corresponding to the highest root of $\mathfrak{e}_{6}$. With the notations of [1], this highest root is $\widetilde{\alpha}=\alpha_{1}+2 \alpha_{3}+3 \alpha_{4}+2 \alpha_{5}+\alpha_{6}+2 \alpha_{2}$. If $h_{\alpha_{i}}$ is the element of the Cartan subalgebra corresponding to the simple root $\alpha_{i}$, it is therefore easy to compute that $\widetilde{\alpha}\left(h_{\alpha_{i}}\right)=0,0,0,1$ for $i=3,4,5,2$. It therefore follows that $x$, considered as an element of the $M$-representation $\mathfrak{n}$, has positive weight, and is not a sum of two positive weights. Therefore, its weight is a fundamental weight of $M \simeq \operatorname{Spin}_{8}$, and since $\operatorname{dim} \mathfrak{n}=8$, we deduce that $\mathfrak{n}$ is either the natural or a spinor representation of $M \simeq \operatorname{Spin}_{8}$. It is thus a consequence of the triality principle which exchanges these three representations that there are three $R$-orbits in $\mathfrak{n}$, of dimensions 0,7 and 8 respectively. This implies that in $\mathbb{P} \mathfrak{n}$ there are only two $R$-orbits.

Let $O$ be the closed $P$-orbit in $P \times^{R} \mathbb{P n}$, which is of codimension 1. The map $\eta$ contracts $O$ to $X_{+}$, and it maps the open $P$-orbit isomorphically to the open $P$-orbit in $\mathbb{P S}_{+}$. By the universal property of blow-ups, we have a morphism $\psi: P \times^{R} \mathbb{P n} \rightarrow$ $B l_{X_{+}}\left(\mathbb{P S}_{+}\right)$, which induces a surjective (thus generically finite) map $\psi_{1}: O \rightarrow \operatorname{Exc}(\phi)$. By Zariski's main theorem, the fibers of $\psi$ (thus also $\psi_{1}$ ) are connected, so $\psi_{1}$ is generically of degree one, which implies that $\operatorname{codim} \operatorname{Exc}(\psi) \geq 2$ if $\operatorname{Exc}(\psi) \neq \emptyset$. On the other hand, $\psi$ is a birational morphism between two smooth varieties, so $\operatorname{Exc}(\psi)$ is either empty or of pure codimension 1. Thus $\operatorname{Exc}(\psi)=\emptyset$ and $\psi$ is an isomorphism. The second claim follows immediately.

Lemma 5. The birational map $G \times{ }^{P} \mathbb{P}(\mathfrak{u}(P)) \rightarrow G \times \times^{Q} \mathbb{P}(\mathfrak{u}(Q))$ is a family of $\mathbb{P}^{5}$ Mukai flops with center $O_{P}$.

Proof : Note that $O_{P}$ has two natural fibrations: $O_{P} \rightarrow G / P$ and $f: O_{P} \rightarrow \mathbb{P} \mathcal{O}_{\min }=$ $G / P_{2}$, where $P_{2}$ is the maximal parabolic associated to the root $\alpha_{2}$. Since moreover $O_{P}$ is closed, this shows that $O_{P}$ is the incidence variety $G /\left(P \cap P_{2}\right)$. Thus $f$ is a locally trivial $P_{2} /\left(P \cap P_{2}\right) \simeq \mathbb{P}^{5}$-bundle. Let $\widetilde{O}_{P}$ be its pre-image in $G \times{ }^{P}(\mathfrak{u}(P)-0)$, 
then $\widetilde{f}: \widetilde{O}_{P} \rightarrow \mathcal{O}_{\text {min }}$ is again a $\mathbb{P}^{5}$-bundle, thus the normal bundle of $\widetilde{O}_{P}$ is isomorphic to $\Omega_{\widetilde{f}}$ (see for example [8], section 3). Now it follows that the normal bundle of $O_{P}$ in $G \times{ }^{P} \mathbb{P}(\mathfrak{u}(P))$ is isomorphic to $\Omega_{f}$. By Lemma 4, the blowing-up of $G \times{ }^{P} \mathbb{P}(\mathfrak{u}(P))$ along $\bar{O}_{P}$ gives $G \times{ }^{R} \mathbb{P} \mathfrak{n}$, while the latter is also the blowing-up of $G \times{ }^{Q} \mathbb{P}(\mathfrak{u}(Q))$ along $O_{Q}$, concluding the proof.

We now give a more geometric proof of theorem 1 in the case $E_{6, I}$ :

Theorem 2. Consider the stratified Mukai flop of type $E_{6, I}, \mu: T_{P} \rightarrow T_{Q}$. Let $B l_{1}\left(T_{P}\right) \rightarrow T_{P}$ (resp. $\left.B l_{1}\left(T_{Q}\right) \rightarrow T_{Q}\right)$ be the blow-up of $T_{P}$ (resp. $T_{Q}$ ) along the zero section and $B l_{1}\left(\bar{T}_{P}^{1}\right)$ (resp. $B l_{1}\left(\bar{T}_{Q}^{1}\right)$ ) the strict transform of the closure of $\widetilde{O}_{P}$ (resp. $\left.\widetilde{O}_{Q}\right)$. Then the birational map $B l_{1}\left(T_{P}\right) \rightarrow B l_{1}\left(T_{Q}\right)$ is a family of $\mathbb{P}^{5}-$ Mukai flops with center $B l_{1}\left(\bar{T}_{P}^{1}\right)$. In particular, there exists a $G$-equivariant isomorphism $\widetilde{\mu}: \widetilde{T}_{P} \simeq \widetilde{T}_{Q}$ which identifies with $\mu$ over the open G-orbits.

Proof : Let $\overline{\mathcal{O}}$ be the nilpotent orbit closure which is resolved by $T_{P}$ and $T_{Q}$. Let $B l_{1}(\overline{\mathcal{O}})$ be the blow-up of $\overline{\mathcal{O}}$ along the origin. By the universal property of blowups, we obtain morphisms $B l_{1}\left(T_{P}\right) \rightarrow B l_{1}(\overline{\mathcal{O}}) \leftarrow B l_{1}\left(T_{Q}\right)$. To simplify the notations, we let $Y_{P}=G \times{ }^{P} \mathbb{P}(\mathfrak{u}(P))$ and $Y_{Q}=G \times^{Q} \mathbb{P}(\mathfrak{u}(Q))$. Then $B l_{1}\left(T_{P}\right)$ (resp. $B l_{1}\left(T_{Q}\right)$, $B l_{1}(\overline{\mathcal{O}})$ ) is just the total space of the tautological line bundle $\mathcal{O}_{Y_{P}}(-1)$ (resp. $\mathcal{O}_{Y_{Q}}(-1)$, $\left.\mathcal{O}_{\mathbb{P} \overline{\mathcal{O}}}(-1)\right)$. The birational map $B l_{1}\left(T_{P}\right) \rightarrow B l_{1}\left(T_{Q}\right)$ is just the pull-back via the morphism $\mathcal{O}_{\mathbb{P} \overline{\mathcal{O}}}(-1) \rightarrow \mathbb{P} \overline{\mathcal{O}}$ of the birational map $Y_{P} \rightarrow Y_{Q}$. Now the claim follows from the precedent lemma.

Now we will relate the variety $\widetilde{T}_{P}$ to the graph closure $Z$ of the flop $\mu$. Let $S \simeq G / R$ denote the closed $G$-orbit in $Z$. By the precedent theorem, $\widetilde{T}_{P}$ gives a resolution of the flop $\mu: T_{P} \rightarrow T_{Q}$, thus there exists a birational morphism $\gamma: \widetilde{T}_{P} \rightarrow Z$.

Corollary 1. The morphism $\gamma: \widetilde{T}_{P} \rightarrow Z$ is isomorphic to the blow-up of $Z$ along $S$.

Proof : Note that $Z$ is smooth, so $\operatorname{Exc}(\gamma)$ is of pure codimension 1. The complementary of the open $G$-orbit in $\widetilde{T}_{P}$ consists of two irreducible divisors: $\widetilde{T}_{P}^{0}$ and $\widetilde{T}_{P}^{1}$. By Lemma 4, $\gamma$ maps $\widetilde{T}_{P}^{1}-\widetilde{T}_{P}^{0}$ isomorphically to its image, which is the blow-up of the 27 -dimensional $G$-orbit in $T_{P}$. This implies that $\operatorname{supp}(\operatorname{Exc}(\gamma))=\widetilde{T}_{P}^{0}$. Now a similar argument as that in the proof of Lemma 4 proves the corollary.

To summarise, the geometric picture of the flop $\mu: T_{P} \rightarrow T_{Q}$ is as follows: the 27 -dimensional $G$-orbit is a $\mathbb{P}^{5}$-bundle over the 22 -dimensional orbit in the nilpotent orbit closure $\overline{\mathcal{O}}$. The flop $\mu$ when restricted to the complementary of the zero section in $T_{P}$ is just a standard Mukai flop of $\mathbb{P}^{5}$-bundles. This restricted flop is resolved by just one blow-up, which is given by $G \times{ }^{R}(\mathfrak{n}-\{0\})$. Thus $\mu$ can be regarded as a degeneration of a 22 -dimensional family of $\mathbb{P}^{5}$-Mukai flops. Note that such a picture is very similar to stratified Mukai flops: $T^{*} G r(2, V) \rightarrow T^{*} G r\left(2, V^{*}\right)$. 


\section{Deformations of stratified Mukai flops}

In this section, we will consider only the $E_{6, I}$ case, which share many analogous properties with the standard Muaki flop (see for example [2]). As before, let $P:=$ $P_{1}$ and $P_{6}$ be the maximal parabolic subgroups of $E_{6}$. They have conjugate Levi subgroups, so we can choose $Q$ in the conjugacy class of $P_{6}$ such that $P$ and $Q$ have the same Levi subgroup $L$. Note that the center $\mathfrak{c}$ of the Lie algebra of $L$ is one-dimensional.

Lemma 6. $\mathfrak{c} \oplus \mathfrak{u}(P)$ is stable under $P$, and the stabilizer of the line $\mathfrak{c}$, considered as an element in $\mathbb{P g}$, is $L$.

Proof : Let $U$ denote the unipotent radical of $P$. According to the Levi decomposition theorem, we have $P=L U$. Therefore, if $p=l u$, and $x \in \mathfrak{c}$, we have $p . x \equiv l . x \equiv x$ modulo $\mathfrak{u}(P)$, proving the first point.

For the second point, denote $\mathfrak{z}(\mathfrak{c})$ the set of elements $z \in \mathfrak{g}$ such that $\forall c \in \mathfrak{c},[z, c]=0$ and $\operatorname{Stab}(\mathfrak{c})=\{g \in G: g \cdot \mathfrak{c} \subset \mathfrak{c}\}$. Note that $\mathfrak{l} \subset \mathfrak{z}(\mathfrak{c})$, and for reason of equal dimension, we have the equality. Therefore, $\operatorname{Stab}(\mathfrak{c}) \subset \operatorname{Stab}(\mathfrak{z}(\mathfrak{c}))=\operatorname{Stab}(\mathfrak{l})$. Now let $\mathfrak{p}$ and $\mathfrak{q}$ be the two parabolic subalgebras containing $\mathfrak{l}$, and let $P, Q$ be the corresponding parabolic subgroups. We have $\operatorname{Stab}(\mathfrak{l}) \subset \operatorname{Stab}(\mathfrak{p}) \cap \operatorname{Stab}(\mathfrak{q})=P \cap Q=L$.

Let $T_{P}=T^{*}(G / P)$ and $T_{Q}=T^{*}(G / Q)$ be the cotangent spaces. We have the onedimensional smooth flat deformations of these two varieties: $E_{P}:=G \times{ }^{P}(\mathfrak{c}+\mathfrak{u}(P))$ and $E_{Q}:=G \times{ }^{Q}(\mathfrak{c}+\mathfrak{u}(Q))$.

Lemma 7. There exists a $G$-equivariant birational map $\psi: E_{P} \rightarrow E_{Q}$ which deforms the Mukai flop $\mu: T_{P} \rightarrow T_{Q}$ to isomorphisms outside the zero fibers.

Proof : Let $z \in \mathfrak{c}$ be a non-zero element. By the previous lemma, its stabilizer in $G$ is $L$ itself. Let $U$ be the unipotent part of $P$, then $P \cdot z=U \cdot z$. Note that $U \cdot z$ is closed and has $z+\mathfrak{u}(P)$ as the tangent space at $z$, which gives $z+\mathfrak{u}(P)=P \cdot z$, thus $G \cdot(z+\mathfrak{u}(P))=G \cdot z$ and $\mathcal{N}:=G \cdot(\mathfrak{c}+\mathfrak{u}(P))=G \cdot(\mathfrak{c}+\mathfrak{u}(Q))$.

We have a natural $G$-equivariant projective morphism $e_{P}: E_{P} \rightarrow \mathcal{N}$. Lemma 6 implies that $e_{P}$ is an isomorphism outside the singular locus of $\overline{\mathcal{O}}:=G \cdot \mathfrak{u}(P)=G \cdot \mathfrak{u}(Q)$, so $e_{P}$ is a small resolution. In a similar way, we have a small resolution $e_{Q}: E_{Q} \rightarrow \mathcal{N}$. Now the claim follows.

Our next task is to resolve this birational map. Recall we have a stratification $T_{P}^{0} \subset \overline{T_{P}^{1}} \subset T_{P}$. We will regard these varieties as subvarieties in $E_{P}$. Let $B l_{1}\left(E_{P}\right)$ be the blow-up of $E_{P}$ along $T_{P}^{0}$ and $\widetilde{E}_{P}$ the blow-up of $B l_{1}\left(E_{P}\right)$ along the strict transform of $\overline{T_{P}^{1}}$. Similarly we can get $\widetilde{E}_{Q}$. The next theorem is analogous to Theorem 1 .

Theorem 3. There exists a unique G-equivariant isomorphism $\widetilde{E}_{P} \simeq \widetilde{E}_{Q}$.

Proof : Let $\mathcal{O}_{1}$ be the 22-dimensional nilpotent orbit in $\overline{\mathcal{O}}$. The pre-image of $\mathcal{O}_{1}$ under the symplectic resolution $\Pi_{P}: T_{P} \rightarrow \overline{\mathcal{O}}$ is a $\mathbb{P}^{5}$-bundle, i.e. $\Pi_{P}^{-1}\left(\mathcal{O}_{1}\right)=\mathbb{P}(F)$ for some vector bundle $\phi: F \rightarrow \mathcal{O}_{1}$ of rank 6 . By our discussions in the precedent section, the variety $\Pi_{Q}^{-1}\left(\mathcal{O}_{1}\right)$ is isomorphic to $\mathbb{P}\left(F^{*}\right)$.

Let $v \in H^{2}\left(T^{*} G / P\right) \simeq H^{2}(G / P)$ be the Kodaira-Spencer class of the deformation $E_{P} \rightarrow \mathfrak{c}$. Note that $v \neq 0$ since the deformation is non-trivial. The Picard group of 
$G / P$ has rank one, thus either $v$ or $-v$ is $\pi_{P}$-ample. In both cases, the restriction of $v$ to a fiber of $\phi$ is non-trivial. By the proof of [8, Lemma 3.6], the normal bundle $\mathcal{N}_{\Pi_{P}^{-1}\left(\mathcal{O}_{1}\right) \mid T_{P}}$ is isomorphic to $\phi^{*}\left(F^{*}\right) \otimes \mathcal{O}_{\phi}(-1)$. Let us denote by $p_{P}: B l_{1}\left(\mathbb{P} E_{P}\right) \rightarrow$ $\mathbb{P} E_{P}$ the blow-up of $\mathbb{P} E_{P}$ along $\mathbb{P} T_{P}^{1}$. Then we have an isomorphism $B l_{1}\left(\mathbb{P} E_{P}\right) \simeq$ $B l_{1}\left(\mathbb{P} E_{Q}\right)$. In particular, the birational map: $\mathbb{P} E_{P} \rightarrow \mathbb{P} E_{Q}$ is a family of $\mathbb{P}^{5}$-standard flops. Now the claim follows from the same arguments of the proof of Theorem 2 .

Remark 2. Another proof of this theorem goes as follows: let $\widetilde{\mathcal{N}} \rightarrow \mathcal{N}$ be the composition of the blow-ups of $\mathcal{N}$ along 0 , then along the strict transform of $\overline{\mathcal{O}}_{\text {min }}$. Then

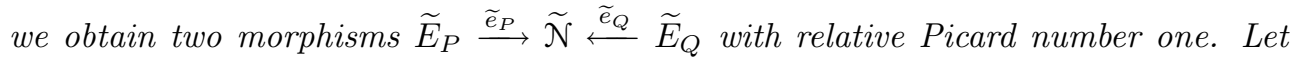
$L$ be an $\widetilde{e}_{P}$-ample line bundle and $L^{\prime}$ its strict transform under the birational map $\widetilde{\psi}: \widetilde{E}_{P} \rightarrow \widetilde{E}_{Q}$. By our theorem in the precedent section, $\widetilde{\psi}$ restricts to an isomorphism on $\widetilde{T}_{P}$, thus $L^{\prime}$ is $\left.\widetilde{e}_{Q}\right|_{\widetilde{T}_{Q}}$-ample. As $\widetilde{e}_{Q}$ is projective with relative Picard number one, we get that $L^{\prime}$ is $\widetilde{e}_{Q}$-ample, thus $\widetilde{\psi}$ is an isomorphism.

\section{Chow groups}

We will denote by $\widetilde{E}$ the variety $\widetilde{E}_{P} \simeq \widetilde{E}_{Q}$. Let $f^{P}: \widetilde{E} \rightarrow E_{P}$ and $f^{Q}: \widetilde{E} \rightarrow E_{Q}$ be the two natural morphisms. Let $\widetilde{T}$ be the central fiber of the family $\widetilde{E} \rightarrow \mathbb{C}$. Note that $\widetilde{T}$ has three irreducible components, one of which is $\widetilde{T}_{P}$. Let $f_{0}^{P}: \widetilde{T} \rightarrow T_{P}$ and $f_{0}^{Q}: \widetilde{T} \rightarrow T_{Q}$ be the natural morphisms. We define two homomorphisms $\Psi=$ $f_{*}^{Q} \circ\left(f^{P}\right)^{*}: C H\left(E_{P}\right) \rightarrow C H\left(E_{Q}\right)$ and $\Psi_{0}=\left(f_{0}^{Q}\right)_{*} \circ\left(f_{0}^{P}\right)^{*}: C H\left(T_{P}\right) \rightarrow C H\left(T_{Q}\right)$. Here $C H(X)$ denotes the Chow ring with integer coefficients of the variety $X$.

Theorem 4. The maps $\Psi, \Psi_{0}$ are both isomorphisms.

Proof : For the proof of the first part, we will follow the proof of Theorem 2.1 in [11], where a similar result is proved for standard flops.

Let $W_{P}$ be any $k$-dimensional sub-variety in $E_{P}$, by Chow's moving Lemma (see for example [7], section 11.4), up to replacing $W_{P}$ by an equivalent cycle, we can assume that $W_{P}$ intersects properly with the cycle $\bar{T}_{P}^{1}+T_{P}^{0}$. Applying twice the blow-up formula (see [7], Theorem 6.7), we obtain that $\left(f^{P}\right)^{*}\left(W_{P}\right)=\widetilde{W}$, where $\widetilde{W}$ is the strict transform of $W_{P}$ under the birational map $f^{P}$, which gives that $\Psi\left(W_{P}\right)=W_{Q}$, where $W_{Q}$ is the strict transform of $W_{P}$ under the birational rational map $\psi: E_{P} \rightarrow E_{Q}$. Remark that $W_{Q}$ intersects no longer properly with the cycle $\bar{T}_{Q}^{1}+T_{Q}^{0}$.

Note that for any irreducible component $C$ of $W_{Q} \cap \bar{T}_{Q}^{1}$, there exists an irreducible component $B$ of the intersection $W_{P} \cap \bar{T}_{P}^{1}$ such that $C \subset e_{Q}^{-1}\left(e_{P}(B)\right)$. Let $\left(f^{Q}\right)^{*} W_{Q}=$ $\widetilde{W}+\Sigma F_{C}$, where $F_{C} \subset\left(f^{Q}\right)^{-1} e_{Q}^{-1} e_{P}(B)$. Note that we have $\operatorname{dim} B=\operatorname{dim} W_{P}-$ $\operatorname{codim} \bar{T}_{P}^{1}$. For a generic point $s \in e_{P}\left(f^{P}\left(F_{C}\right)\right)$, we have

$$
\operatorname{dim} F_{C, s}=\operatorname{dim} F_{C}-\operatorname{dim} e_{P}(B) \geq \operatorname{dim} W_{P}-\operatorname{dim} B=\operatorname{codim} \bar{T}_{P}^{1}=6 .
$$


However a generic fiber of $\left.e_{P}\right|_{\bar{T}_{P}^{1}}$ is 5-dimensional, thus $F_{C, s}$ contains positive dimensional fibers of $f^{P}$, thus $f_{*}^{P}\left(F_{C}\right)=0$, which gives that $f_{*}^{P}\left(f^{Q}\right)^{*}$ is an inverse of $\Psi$, thus the claim of the first part.

Let $i_{P}: T_{P} \rightarrow E_{P}, i_{Q}: T_{Q} \rightarrow E_{Q}$ and $i: \widetilde{T} \rightarrow \widetilde{E}$ be the natural inclusions. The notations are summarized in the following diagram:

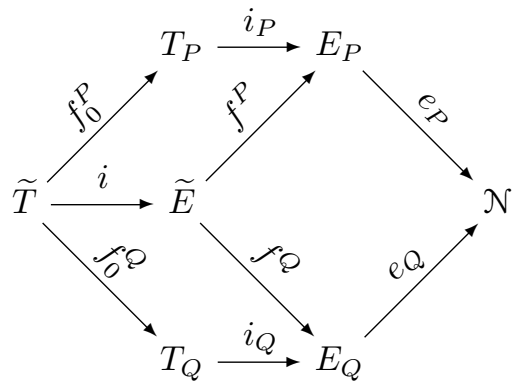

We have the following diagrams:

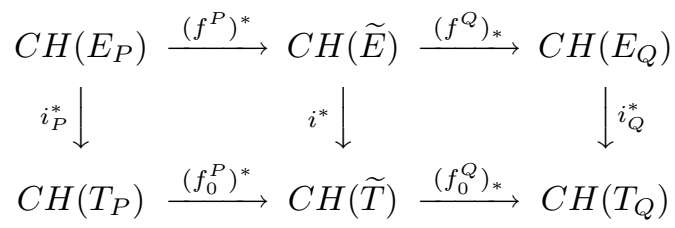

The first diagram is commutative since $f^{P} \circ i=i_{P} \circ f_{0}^{P}: \widetilde{T} \rightarrow E_{P}$. Now we show that the second diagram is also commutative. In fact, for any $[Y] \in C H_{k}(\widetilde{E})$, we may assume it intersects properly with $\widetilde{T}$ by using Chow's moving lemma. Note that $f^{Q}$ is an isomorphism outside $\widetilde{T}$, thus $\left(f^{Q}\right)_{*}[Y]=\left[f^{Q}(Y)\right]_{k}$. It follows that $i_{Q}^{*}\left(f^{Q}\right)_{*}[Y]=\left[f^{Q}(Y) \cap T_{Q}\right]_{k}$. Similarly we have $\left(f_{0}^{Q}\right)_{*} i^{*}[Y]=\left[f_{0}^{Q}(\widetilde{T} \cap Y)\right]_{k}$, which gives $\left(f_{0}^{Q}\right)_{*} i^{*}=i_{Q}^{*}\left(f^{Q}\right)_{*}$. It follows that $i_{Q}^{*} \circ \Psi=\Psi_{0} \circ i_{P}^{*}$. Now the claim follows from the fact that both $i_{Q}^{*}$ and $i_{P}^{*}$ are both isomorphisms.

Remark 3. (i). As shown in [11], this also proves that $\Psi$ (resp. $\left.\Psi_{0}\right)$ induces an equivalence between the motives $\left[E_{P}\right]$ and $\left[E_{Q}\right]$ (resp. $\left[T_{P}\right]$ and $\left[T_{Q}\right]$ ).

(ii). The same proof also works for stratified Mukai flops such as $T^{*} G r(2, V) \rightarrow$ $T^{*} \operatorname{Gr}\left(2, V^{*}\right)$, which has been proved using a different method by Namikawa ([13]) on the level of K-groups. His proof morally works for stratified Mukai flops of type D. So the only unsolved case is the stratified Mukai flops of type $E_{6, I I}$.

Example 1. To simplify the notations, we denote by $b_{P}: B_{P} \rightarrow T_{P}$ the blow-up along $X_{P}$ and $F_{P}$ its exceptional divisor. Let $\Sigma_{P} \subset T_{P}$ be the closure of the 27dimensional $G$-orbit and $\widetilde{\Sigma}_{P}$ its proper transform under $b_{P}$. Let $h_{P}: \widetilde{T}_{P} \rightarrow B_{P}$ the blow-up along $\widetilde{\Sigma}_{P}$. Similar notations will be used on the dual-side. Finally let

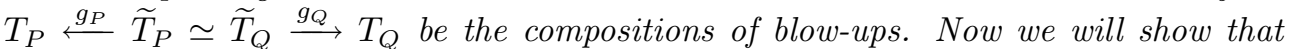
the naturally defined morphism $\Phi:=\left(g_{Q}\right)_{*} \circ\left(g_{P}\right)^{*}: C H\left(T_{P}\right) \rightarrow C H\left(T_{Q}\right)$ is not an isomorphism. Note that this situation is very similar to that of usual Mukai flops (see for example [13, section 2], [11, section 6]). 
By the blow-up formula, we have $\left(b_{P}\right)^{*}\left(\Sigma_{P}\right)=\widetilde{\Sigma}_{P}+\Gamma_{P}$, where $\Gamma_{P}$ is a cycle supported on $F_{P}$. Using Chow's moving lemma (with ambient space $F_{P}$ ), we can assume that $\Gamma_{P}$ intersects properly with $F_{P} \cap \widetilde{\Sigma}_{P}$. Then $\gamma:=\left(h_{Q}\right)_{*} \circ\left(h_{P}\right)^{*} \Gamma_{P}$ will be a cycle supported on $F_{Q}$. Note that $b_{Q}\left(F_{Q}\right)$ is 16-dimensional, so $\left(b_{Q}\right)_{*} \gamma=0$. Note that the birational map $B_{P} \rightarrow B_{Q}$ is a family of $\mathbb{P}^{5}$ Mukai flops. One deduces that (see Example 6.5 [11]) that $\left(h_{Q}\right)_{*} \circ\left(g_{P}\right)^{*}\left(\Sigma_{P}\right)=5 \widetilde{\Sigma}_{Q}+\gamma$, which gives that $\Phi\left(\Sigma_{P}\right)=5 \Sigma_{Q}$. Similarly for $\Phi^{\prime}:=\left(g_{P}\right)_{*} \circ\left(g_{Q}\right)^{*}: C H\left(T_{Q}\right) \rightarrow C H\left(T_{P}\right)$, we get $\Phi^{\prime}\left(\Sigma_{Q}\right)=5 \Sigma_{P}$. Thus $\Phi$ and $\Phi^{\prime}$ are not isomorphisms if $\Sigma_{P}$ is non-zero in $C H^{5}\left(T_{P}\right)$. This can be seen as follows.

Let $q: \mathbb{P}\left(T^{*} X_{P} \oplus \mathcal{O}\right) \rightarrow X_{P}$ be the natural projection and $\bar{\Sigma}_{P}$ the closure of $\Sigma_{P}$. By [7, Prop. 3.3], we have $\left(\pi_{P}^{*}\right)^{-1}\left(\Sigma_{P}\right)=q_{*}\left(c_{16}(\xi) \cap \bar{\Sigma}_{P}\right)$, where $\xi$ is the universal quotient bundle (of rank 16) of $q^{*}\left(T^{*}\left(X_{P}\right) \oplus \mathcal{O}\right)$. Note that $c_{16}(\xi)=\sum_{i=0}^{16} c_{1}(\mathcal{O}(1))^{i} \cap$ $c_{16-i}\left(q^{*} T^{*} X_{P}\right)$. Thus we get $\left(\pi_{P}^{*}\right)^{-1}\left(\Sigma_{P}\right)=\sum_{i=0}^{16} q_{*}\left(c_{16-i}\left(q^{*} T^{*} X_{P}\right) \cap c_{1}(\mathcal{O}(1))^{i} \cap\right.$ $\left.\bar{\Sigma}_{P}\right)=\sum_{i=0}^{16} c_{16-i}\left(T^{*} X_{P}\right) \cap q_{*}\left(c_{1}(\mathcal{O}(1))^{i} \cap \bar{\Sigma}_{P}\right)$. For reason of dimension, $q_{*}\left(c_{1}(\mathcal{O}(1))^{i} \cap\right.$ $\left.\bar{\Sigma}_{P}\right)=0$ if $i \neq 5$, and $q_{*}\left(c_{1}(\mathcal{O}(1))^{5} \cap \bar{\Sigma}_{P}\right)=d[X / P]$, where $d$ is the degree of the closure of $\widehat{X}_{+}$. From this, we deduce that $\left(\pi_{P}^{*}\right)^{-1}\left(\Sigma_{P}\right)=d c_{5}\left(T^{*} X_{P}\right)$, which is non-zero since $c_{5}\left(T^{*} X_{P}\right) \neq 0$ (this can be deduced for example from the calculus made in [10, section 7$])$.

As pointed out by $M$. Brion, for any rational homogeneous variety $Z$ of dimension $n$, the classes $c_{i}\left(T^{*} Z\right)$ are never zero when $0 \leq i \leq n$. In fact, if $c_{i}\left(T^{*} Z\right)=0=$ $c_{i}(T Z)$, then by [7, Example 12.1.8], there exists a sub-bundle $F$ of $T Z$ isomorphic to the trivial bundle such that the rank of the quotient $T Z / F i s<i$. On the other hand, $T Z$ has no trivial sub-bundles, since every vector field on $Z$ has a zero.

Example 2. Let $Z$ be the graph of the flop $T_{P} \rightarrow T_{Q}$ and $T_{P} \stackrel{q_{1}}{\longleftarrow} Z \stackrel{q_{2}}{\longrightarrow} T_{Q}$ the two graph projections. Let $\gamma: \widetilde{T}_{P} \rightarrow Z$ be the natural morphism, which is a blowup along a smooth subvariety (Corollary 1). By proposition 6.7 (b) ([7]), the map $\gamma_{*} \circ \gamma^{*}: C H(Z) \rightarrow C H(Z)$ is the identity.

Now consider the morphism $\Gamma:=\left(q_{2}\right)_{*} \circ q_{1}^{*}: C H\left(T_{P}\right) \rightarrow C H\left(T_{Q}\right)$. Then $\Gamma=$ $\left(q_{2}\right)_{*} \circ \gamma_{*} \circ \gamma^{*} \circ q_{1}^{*}=\Phi$. Thus the morphism $\Gamma$ induced by the graph of the flop does not give an isomorphism.

\section{Acknowledgements}

We are grateful to E. Markman for his clear explanations on [12]. The second named author would like to thank Y. Namikawa for helpful discussions and the Max Planck Institute (Bonn) for the hospitality.

\section{References}

[1] N. Bourbaki, Groupes et algèbres de Lie, chapitre 6, Hermann (1968).

[2] P.-E. Chaput, On Mukai flops for Scorza varieties, preprint. Math.AG/0601734.

[3] C. Chevalley, The algebraic theory of spinors and Clifford algebras, Collected works. Vol. 2, Springer-Verlag, Berlin (1997).

[4] C. D. Concini and C. Procesi, Complete symmetric varieties, in Invariant theory (Montecatini, 1982), Lecture Notes in Mathematics 996 (1983) 1-44.

[5] J. R. Faulkner, Octonion planes defined by quadratic Jordan algebras, Memoirs of the American Mathematical Society 104 (1970) 
[6] B. Fu, Extremal contractions, stratified Mukai flops and Springer maps, Adv. Math. 213 (2007) 165-182.

[7] W. Fulton, Intersection theory, Ergebnisse der Mathematik und ihrer Grenzgebiete (3) 2., Springer-Verlag, Berlin (1984).

[8] D. Huybrechts, Birational symplectic manifolds and their deformations, J. Differential Geom. 45 (1997) 488-513.

[9] J. Igusa, A classification of spinors up to dimension twelve, Amer. J. Math. 92 (1970) 997-1028.

[10] A. Iliev and L. Manivel, The Chow ring of the Cayley plane, Compos. Math. 141 (2005) 146-160.

[11] Y.-P. Lee, H.-W. Lin, and C.-L. Wang, Flops, motives and invariance of quantum rings, Annals of Math. (to appear). Math.AG/0608370.

[12] E. Markman, Brill-Noether duality for moduli spaces of sheaves on K3 surfaces, J. Algebraic Geom. 10 (2001) 623-694.

[13] Y. Namikawa, Mukai flops and derived categories. II, in Algebraic structures and moduli spaces, Vol. 38 of CRM Proc. Lecture Notes, 149-175, Amer. Math. Soc., Providence, RI (2004).

[14] - Birational geometry of symplectic resolutions of nilpotent orbits, in Moduli spaces and arithmetic geometry, Vol. 45 of Adv. Stud. Pure Math., 75-116, Math. Soc. Japan, Tokyo (2006).

[15] M. Thaddeus, Complete collineations revisited, Math. Ann. 315 (1999) 469-495.

Labo. J. Leray, Faculté des sciences, Université de NANTES, 2, Rue de la Houssinière, BP 92208 F-44322 Nantes Cedex 03 - France

E-mail address: chaput@math.univ-nantes.fr

E-mail address: fu@math.univ-nantes.fr 